\title{
Monte Carlo studies of ohmic hole mobility in silicon and germanium: Examination of the optical phonon deformation potential
}

\author{
J. M. Hinckley and J. Singh \\ Department of Electrical Engineering and Computer Science, The University of Michigan, Ann Arbor, \\ Michigan 48109-2122
}

(Received 28 March 1994; accepted for publication 17 June 1994)

\begin{abstract}
Monte Carlo methods which have been widely used for studying high field electron and hole transport, so far have not been applied to the complex problem of Ohmic hole transport. We present a versatile generalization of the Monte Carlo approach for Ohmic hole mobility studies and apply it to pure silicon and germanium. In particular, we examine the role of the optical phonon deformation potential $d_{0}$ in controlling the temperature dependence of the mobility.
\end{abstract}

\section{INTRODUCTION}

Transport phenomena have now been theoretically studied in silicon and germanium, for four decades. Generally, conduction-band and valence-band phenomena have received different treatments. Although the conduction-band edges of both materials consist of multiple valleys, these are separated from one another in $k$-space. Thus, there has been no particular difficulty in understanding electron transport phenomena, as an extension of single valley transport. Theoretical treatment of electron transport has been straightforward and is developed to a high degree of precision. Hole transport, however, is fundamentally complex, due to a multiple degeneracy in the valence band at $k=0$, which is only partially lifted by spin-orbit coupling. The intrinsic interband coupling and anisotropies have made theoretical treatment nonstraightforward. This has compelled most theoretical treatments to make such approximations that their results are of questionable precision.

Precise modeling of hole transport requires taking into account the $p$-like symmetry of the valence-band states. This symmetry is expressed in the three band $k \cdot p$ Hamiltonian, presented in the next section, which is appropriate for states near the top of the valence band in a semiconductor whose band gap is somewhat larger that the spin-orbit splitting. Transport theories are, in significant part, based on the models of the scattering processes involved. In pure covalent semiconductors, such as silicon and germanium, the primary scattering models are those for deformation potential acoustic phonon scattering and deformation potential optical phonon scattering, as stated above. Fundamental to these, is a deformation potential theory for the valence band, which accounts for the $p$-like symmetry of the valence band. Pikus and $\mathrm{Bir}^{1,2}$ have developed such a theory, which involves four independent deformation potentials: $a$, related to hydrostatic compression, $b$, related to uniaxial strain along the [100] axis, $d$, related to uniaxial strain along the [111] axis, and $d_{0}$, related to deformations associated with optical phonons. Pikus and $\mathrm{Bir}^{2,3}$ and Tiersten ${ }^{4,5}$ have developed a model of hole-acoustic phonon scattering based on this deformation potential theory. Pikus and $\mathrm{Bir}^{2,3}$ Lawaetz, $^{6}$ and Szmulowicz ${ }^{7}$ have developed successive refinements to a model of hole-optical phonon scattering, again reflecting the $p$-like symmetry of the valence-band states.
Aside from the formal precision of a transport theory based on scattering models with the correct symmetry, the advantage of using the above models in transport calculations is that the deformation potentials associated with acoustic phonon scattering, $a, b$, and $d$, can be determined from methods apart from a transport calculation fitting. In other words, the parameters for acoustic phonon scattering need not be treated as phenomenological fitting parameters in a transport study, but can be input as known parameters in the same way that the lattice constant and the mass density are. Even' the deformation potential $d_{0}$ associated with optical phonon scattering could, in principle, be independently determined. ${ }^{8}$ However, practically, $d_{0}$ is treated as a phenomenological parameter, which is adjusted to fit the transport calculations to known measurements, but, it is only this one, single parameter that is treated as adjustable, thus increasing the precision of the theory.

In the last decade, Madarasz and Szmulowicz have advanced and used these scattering models in very careful calculations of hole transport in silicon and in germanium. ${ }^{7,9-14}$ Their method involves solving the Boltzmann transport equation by performing a harmonic expansion on the distribution function and solving for the first four odd-ordered moments, thus going significantly beyond the relaxation time approximation. The method allows determination of very low field transport properties, such as conduction mobility and Hall mobility. Their method is definitive in this area. Szmulowicz was able to fit measured mobilities in pure silicon and germanium over a broad temperature range, using only a single, fit value of $d_{0}$ in each material. ${ }^{7}$

More recently, Hinckley and Singh ${ }^{15,16}$ have developed an alternative, Monte Carlo hole transport calculation method, based on the same scattering models as refined and used by Madarasz and Szmulowicz. This method is applicable to studies of zero-field carrier dynamics and high-field transport, although not applicable to low-field transport and magnetotransport. The high-field capability of the Monte Carlo method complements the low-field capability of Madarasz and Szmulowicz' method. An overlap exists in the area of Ohmic conduction. The Madarasz and Szmulowicz method can directly yield the conduction mobility, whereas the Hinckley and Singh Monte Carlo method can give the zero-field diffusion coefficient, which is then related to the conduction mobility through the Einstein relation. 
Using the three-band $k \cdot p$ band structure and the Pikus and $\mathrm{Bir}$ deformation potential theory, the Madarasz and Szmulowicz, and the Hinckley and Singh hole transport methods have advanced the precision of transport calculations to the point where the accuracy of the results is primarily limited by the variation in published material parameters and measured mobilities. Various values may be found in the literature for the band structure parameters, the elastic stiffness constants, and the valence-band deformation potentials $a, b$, and $d$, as well as for the room-temperature mobility and the temperature coefficients of the mobility for both silicon and germanium.

The purpose of this work is to extend the Monte Carlo method so that it can be applied to calculations of Ohmic hole mobility and to determine the best values of the optical phonon deformation potential $d_{0}$ in both silicon and germanium over a range of published material parameters, and to examine how the best $d_{0}$ varies with a variation in the other parameters. The best $d_{0}$ is determined to be that which gives a calculated room-temperature mobility agreeing with a published measured value. Given a single fitting parameter $d_{0)}$ and a single datum to fit to, $\mu(300 \mathrm{~K})$, an agreement is generally achievable, no matter which material parameters are used. Therefore, we have also examined the temperature dependence of the calculated mobility, using the best values of $d_{0}$, for each material parameter set, in both semiconductors. This is done to determine which material parameter set, along with the best $d_{0}$ for that set, could be used to most accurately model transport in each material.

The content of the rest of this paper is as follows. The next section describes the modeling used, both the band structure and scattering models and the Monte Carlo implementation which accounts for the complexities of the valence band. Section III briefly discusses the various material parameter values and mobilities found in the published literature for silicon and germanium. These are the relevant parameters for input to the transport calculation, then the calculation procedure for determining the transport properties is described. Section IV gives the results of the calculations. Section V contains a discussion of the results and presents the conclusions.

\section{MODEL}

The models of the band structure and scattering rates as well as the Monte Carlo implementation used in this work are described in substantial detail in separate publications. ${ }^{15-17}$ The presentation given here will cover only briefly the main aspects of the models and methods used.

\section{A. Band structure}

The valence band structure is calculated using a three band $k \cdot p$ method, with spin-orbit coupling. The eigenstates are formed from the six-dimensional basis: $|x \uparrow\rangle,|y \uparrow\rangle,|z \uparrow\rangle,|x \downarrow\rangle,|y \downarrow\rangle,|z \downarrow\rangle$. The band structure is obtained by calculating the eigenvalues of $H_{k p}+H_{s o}$. The $k \cdot p$ Hamiltonian matrix is: ${ }^{18}$

$$
H_{k p}=\left[\begin{array}{cc}
H^{\prime} & 0_{3 \times 3} \\
0_{3 \times 3} & H^{\prime}
\end{array}\right] \uparrow
$$

$$
H^{\prime}=-\frac{\hbar^{2}}{2 m}\left[\begin{array}{ccc}
L k_{x}^{2}+M\left(k_{y}^{2}+k_{z}^{2}\right) & N k_{x} k_{y} & N k_{z} k_{x} \\
N k_{x} k_{y} & L k_{y}^{2}+M\left(k_{z}^{2}+k_{x}^{2}\right) & N k_{y} k_{z} \\
N k_{z} k_{x} & N k_{y} k_{z} & L k_{z}^{2}+M\left(k_{x}^{2}+k_{y}^{2}\right)
\end{array}\right] \begin{aligned}
& x \\
& y \\
& z
\end{aligned}
$$

where $m$ is the free electron mass. The dimensionless terms $L, M$, and $N$ are related to the Luttinger $\gamma$ parameters: ${ }^{19}$

$$
L=\gamma_{1}+4 \gamma_{2}, \quad M=\gamma_{1}-2 \gamma_{2}, \quad N=6 \gamma_{3} .
$$

The spin-orbit interaction is included by adding $H_{s o}$ to $H_{k p}$. In the above basis, $H_{s o}$ is: $^{4}$

$$
H_{s o}=\frac{\Delta_{0}}{3}\left[\begin{array}{rrrrrr}
0 & -i & 0 & 0 & 0 & 1 \\
i & 0 & 0 & 0 & 0 & -i \\
0 & 0 & 0 & -1 & i & 0 \\
0 & 0 & -1 & 0 & i & 0 \\
0 & 0 & -i & -i & 0 & 0 \\
1 & i & 0 & 0 & 0 & 0
\end{array}\right] \begin{gathered}
x \uparrow \\
z \uparrow \\
x \downarrow \\
y \downarrow \\
z \downarrow
\end{gathered}
$$

where $\Delta_{0}$ is the zone center $(k=0)$ spin-orbit splitting.

\section{B. Scattering processes}

Two mechanisms of lattice scattering are used in this work: deformation potential acoustic phonon scattering and deformation potential (nonpolar) optical phonon scattering. Each scattering mechanism may drive both inter- and intraband transitions between and within the three valence bands. This results in three intraband and six interband modes of scattering for each mechanism.

Scattering rates for transitions from band $n$ (i.e., $\mathrm{HH}$, $\mathrm{LH}$, or $\mathrm{SH}$ ) to band $n^{\prime}$ (i.e., $\mathrm{HH}, \mathrm{LH}$, or $\mathrm{SH}$ ) by mechanism $m$ (i.e., acoustic phonon or optical phonon), are calculated using Fermi's second Golden Rule

$$
\begin{aligned}
W_{m ; n, n^{\prime}}(\mathbf{k})= & \frac{V_{c}}{(2 \pi)^{3}} \frac{2 \pi}{\hbar} \int d^{3} k^{\prime}\left|M_{m ; n, n^{\prime}}\left(\mathbf{k}, \mathbf{k}^{\prime}\right)\right|^{2} \\
& \times \delta\left(E_{n}+\Delta E_{m}-E^{\prime}{ }_{n^{\prime}}\right),
\end{aligned}
$$

where $M$ is the scattering matrix element, $V_{c}$ is the crystal 
volume, and the density of final states is given by the delta function. Conservation of energy reduces this integral from three to two dimensions, with the integration being carried out over the constant energy surface $E=E_{n^{\prime}}^{\prime}$. Because of the complex warped nature of the valence band constant en- ergy surfaces, the integration is done numerically. We have found a ten-point Newton-Cotes algorithm to work satisfactorily for the integration of each dimension separately.

The squared modulus of the scattering matrix element for acoustic phonon scattering is: ${ }^{\text {? }}$

$$
\left|M_{\mathrm{ac} ; n, n^{\prime}}\left(\mathbf{k}, \mathbf{k}^{\prime}\right)\right|^{2}=\frac{k_{B} T}{\rho V_{c}} \sum_{/=1}^{3} \frac{1}{s^{2}}\left|\sum_{\alpha, \beta=1}^{3} \hat{q}_{\alpha}\left(\sum_{j, j^{\prime}=1}^{3}\left\langle\psi_{n^{\prime}}\left(\mathbf{k}^{\prime}\right) \mid j^{\prime}\right\rangle D_{\alpha \beta}^{j^{\prime} j}\left\langle j \mid \psi_{n}(\mathbf{k})\right\rangle\right) e_{\beta}^{\prime}(\mathbf{q})\right|^{2},
$$

where $k_{B} T \quad$ is Boltzmann's constant times temperature,

$\rho \quad$ is the mass density,

$V_{c} \quad$ is the crystal volume,

$s, \quad$ is the phonon speed in mode $/$,

$\hat{q}_{\alpha}$. is the ath direction cosine of the phonon wave vector,

$e_{\beta}^{\prime}(\mathbf{q}) \quad$ is the $\beta$ th component of the phonon polarization vector in mode $\zeta$ for a phonon with wave vector $\mathbf{q}$,

$D_{\alpha \beta}^{j^{\prime} j} \quad$ is the $j^{\prime}, j$ element of the $\alpha, \beta$ deformation potential operator,

$\left\langle j \mid \psi_{n}(\mathbf{k})\right\rangle$ is the projection of the initial wave function onto $|x\rangle(j=1),|y\rangle(j=2)$ or $|z\rangle(j=3)$.

The sum over $/$ is a sum over the three phonon polarizations. A factor of 2 is included, to account for both emission and absorption at once. The operators $D_{\alpha \beta}$ are given as $3 \times 3$ matrices in the $x, y, z$ basis:

$$
\begin{aligned}
& D_{x x}=\left[\begin{array}{ccc}
f & 0 & 0 \\
0 & m & 0 \\
0 & 0 & m
\end{array}\right] \quad D_{y y}=\left[\begin{array}{ccc}
m & 0 & 0 \\
0 & \nearrow & 0 \\
0 & 0 & m
\end{array}\right] \begin{array}{l}
x \\
y \\
z
\end{array} \\
& D_{z z}=\left[\begin{array}{ccc}
m & 0 & 0 \\
0 & m & 0 \\
0 & 0 & f
\end{array}\right] \quad D_{z x}=\left[\begin{array}{ccc}
0 & 0 & \frac{n}{2} \\
0 & 0 & 0 \\
\frac{n}{2} & 0 & 0
\end{array}\right] \begin{array}{l}
x \\
y \\
z
\end{array} \\
& D_{x y}=\left[\begin{array}{ccc}
0 & \frac{n}{2} & 0 \\
\frac{n}{2} & 0 & 0 \\
0 & 0 & 0
\end{array}\right] \quad D_{y z}=\left[\begin{array}{ccc}
0 & 0 & 0 \\
0 & 0 & \frac{n}{2} \\
0 & \frac{n}{2} & 0
\end{array}\right]^{x} y
\end{aligned}
$$

and $D_{x y}=D_{y x}, D_{y z}=D_{z y}, D_{z x}=D_{x z}$. The valence-band deformation potentials $l, m$, and $n$ are related to the more familiar $a, b$, and $d$ by

$$
\ell=a+2 b, \quad m=a-b, \quad n=\sqrt{3} d .
$$

The usual approach to hole-acoustic phonon scattering ${ }^{20,21}$ is formally equivalent to setting $n=0$ and replacing $\ell$ and $m$ with an avcraged effective acoustic phonon scattcring deformation potential, $\Xi_{a}$, and replacing $s$, with an averaged sound speed. The present model does not make this approxi- mation and retains the full nondiagonal form of the deformation potential operators $D_{\alpha \beta}$. The phonon polarization vectors which are used are those published in Refs. 9 and 22. The longitudinal and transverse phonon speeds are obtained from spherically averaged elastic stiffness tensor elements, as published in Ref. 9.

This model treats the scattering as elastic and uses equipartition of phonon modes, both of which are valid approximations at room temperature. However, since this work involves lower temperature calculations as well, these two approximations should be quantitatively verified. At a given temperature, the average energy of acoustic phonons involved in scattering is $\left\langle e_{p}\right\rangle=\hbar\langle\omega\rangle=\hbar v_{s}\langle q\rangle$, where $v_{s}$ is the average sound speed and $\langle q\rangle$ is the average phonon wave vector magnitude. Conservation of momentum during scattering requires that $q$ lies between 0 and $2 k$, where $k$ is the magnitude of the hole wave vector. On the average, $\langle q\rangle$ $=\langle k\rangle$, where $\langle k\rangle$ is the average hole wave vector at the given temperature. This may be related to the average hole energy, through the approximate relation

$$
\langle E\rangle=\frac{\hbar^{2}\langle k\rangle^{2}}{2 m^{*}},
$$

in which the warping of the hole energy surfaces has been accounted for in the value of $m^{*}$. Thus the average energy of acoustic phonons involved in scattering holes is

$$
\begin{aligned}
\left\langle e_{p}\right\rangle & =v_{s} \sqrt{2 m^{*}\langle E\rangle}=0.50 \sqrt{\frac{\langle E\rangle}{\mathrm{meV}}} \mathrm{meV} \\
& =0.22 \sqrt{\frac{\langle E\rangle}{\mathrm{meV}}} \mathrm{meV}(\mathrm{Ge}) .
\end{aligned}
$$

Anticipating the results of the calculations to be presented later, the average hole energies are $\langle E\rangle=16.5 \mathrm{meV}$ and 43.7 $\mathrm{meV}$ in Si at $T=150 \mathrm{~K}$ and $300 \mathrm{~K}$, respectively, and $\langle E\rangle$ $=10.0 \mathrm{meV}$ and $41.5 \mathrm{meV}$ in $\mathrm{Ge}$ at $T=100 \mathrm{~K}$ and $300 \mathrm{~K}$, respectively. The temperature values presented here are the limits of the temperature ranges, in each material, over which the mobility data are fit (see Sec. IV). The ratio of average 
hole energy to average acoustic phonon energy is $\langle E\rangle /\left\langle e_{p}\right\rangle=8.2$ and 13 in Si at $T=150 \mathrm{~K}$ and $300 \mathrm{~K}$, respectively, and $\langle E\rangle /\left\langle e_{p}\right\rangle=14$ and 29 in Ge at $T=100 \mathrm{~K}$ and 300 $\mathrm{K}$, respectively. In the worst case of $\mathrm{Si}$ at $T=150 \mathrm{~K}$, elastic acoustic phonon scattering appears to be a fair approximation. The average occupation number of acoustic phonons involved in hole scattering is $N=1 /\left[\exp \left(\left\langle e_{p}\right\rangle / k_{B} T\right)-1\right]=5.9$ and 7.4 in Si at $T=150$ and $300 \mathrm{~K}$, respectively, and 12 and 17 in $\mathrm{Ge}$ at $T=100$ and $300 \mathrm{~K}$, respectively. Comparing $N+(N+1)$ with $2 k_{B} T /\left\langle e_{p}\right\rangle$, a difference of no more than $0.2 \%$ is found, over the same temperature ranges as above. Thus, equipartition is also a valid approximation.

The squared modulus of the scattering matrix element for optical phonon scattering is: ${ }^{7}$

$$
\left|M_{\mathrm{op} ; n, n^{\prime}}\left(\mathbf{k}, \mathbf{k}^{\prime}\right)\right|^{2}=d_{0}^{2} \frac{3 \hbar\left(n_{0}+\frac{1}{2} \mp \frac{1}{2}\right)}{\rho a_{0}^{2} \omega_{0} V_{c}} \sum_{/=1}^{3}\left|\sum_{j, j^{\prime}=1}^{3}\left\langle\psi_{n^{\prime}}\left(\mathbf{k}^{\prime}\right) \mid j^{\prime}\right\rangle A_{/}^{j^{\prime} j}\left\langle j \mid \psi_{n}(\mathbf{k})\right\rangle\right|^{2},
$$

where $d_{0}$ is the optical phonon deformation potential,

$n_{0}$ is the optical phonon mean occupation number; $n_{0}+\frac{1}{2}-\frac{1}{2}=n_{0}$ : phonon absorption, $n_{0}+\frac{1}{2}+\frac{1}{2}=n_{0}+1:$ phonon emission,

$\rho$ is the mass density,

$a_{0}$ is the lattice constant,

$\omega_{0}$ is the zone center optical phonon frequency.

$V_{c}$ is the crystal volume,

$A_{f}^{j^{\prime} j}$ is the $j^{\prime}, j$ element of the permutation operator corresponding to the $/$ th optical phonon mode.

Again, the sum over $\zeta$ is a sum over the three phonon polarizations. The operators $A$, are determined by the symmetry of the lattice, ${ }^{7}$ and are given as $3 \times 3$ matrices in the $x, y, z$ basis:

$$
\begin{aligned}
& A_{x}=\left[\begin{array}{lll}
0 & 0 & 0 \\
0 & 0 & 1 \\
0 & 1 & 0
\end{array}\right] \quad A_{y}=\left[\begin{array}{lll}
0 & 0 & 1 \\
0 & 0 & 0 \\
1 & 0 & 0
\end{array}\right] \begin{array}{l}
x \\
y \\
z
\end{array} \\
& A_{z}=\left[\begin{array}{lll}
0 & 1 & 0 \\
1 & 0 & 0 \\
0 & 0 & 0
\end{array}\right] x \\
& y
\end{aligned}
$$

In addition to being a function of energy, each scattering rate is anisotropic and its value will depend upon the orientation of the initial trajectory, $\mathbf{k}$, with respect to the crystallographic axes.

\section{Monte Carlo implementation}

The Monte Carlo method used in this work is an implementation of the fundamental algorithm most commonly used in classical transport simulations. The transport of a particle is simulated as a great number of repetitions of the basic cycle of free flight under any imposed fields, then scattering by one of several mechanisms, followed by a determination of the new post-scattering state, then return for another free flight, beginning the next cycle.

The special features of this Monte Carlo method are associated with the handling of the various anisotropies of the band structure and scattering mechanisms. These fall into three areas: particle energy, scattering rates, and determination of the post-scattering trajectory.

During the operation of the Monte Carlo, energies are obtained by solving for the eigenvalues of $H_{s o}+H_{k p}$. [Eqs. (2) and (3)]. This gives accurate values of the energy, which is very important for determining correct particle motion during free flight.

Like energies, scattering rates are anisotropic, exhibiting cubic symmetry with respect to variations in the initial trajectory. Calculation of the scattering rates involves a twodimensional numerical integration that is too time consuming, however, to be done practically during the Monte Carlo operation. The scattering rates, therefore, must be calculated beforehand and input in tabulated form to the Monte Carlo. The tabulation consists of values obtained over a mesh in energy, for an assortment of initial trajectories. The assortment of initial trajectories may be considered to constitute an additional two-dimensional mesh, resulting in an overall 3D mesh on which the scattering rates are calculated.

The design of the mesh is one of the critical aspects of the Monte Carlo implementation and therefore merits a full description. An energy mesh with variable spacing is used, which is constructed in the following way.

(1) A mesh point is taken at $0 \mathrm{eV}$.

(2) An upper limit of energy is chosen, typically $0.5 \mathrm{eV}$, where another mesh point is taken. These two points establish the ends of the mesh.

(3) Next, a mesh point is taken at the energy of scattering threshold. Each specific choice of initial band, final band, and scattering mechanism will have its own threshold. For example, within the heavy hole band, (elastic) acoustic phonon scattering will have a threshold of $0 \mathrm{eV}$, whereas, scattering from the heavy hole band to the splitoff band by optical phonon emission will have a threshold which is the sum of the spin-orbit splitting and the energy of an optical phonon $(0.0440 \mathrm{eV}+0.0628 \mathrm{eV}=0.1068 \mathrm{eV}$ in the case of silicon).

(4) Two points are taken on either side of the threshold. Typically, a very small distance of $0.0001 \mathrm{eV}$ is chosen.

(5) Then, a finely pitched mesh is taken, starting from the threshold, and continuing for a specified number of steps. Typical values are a pitch of $0.001 \mathrm{eV}$ and 20 steps. 
(6) Finally, a more coarsely pitched mesh is taken, starting at the high energy end of the grid and working down in energy until the finely pitched mesh is reached. A typical pitch of this mesh is $0.02 \mathrm{eV}$.

It will be seen that the scattering rate for each specific choice of initial band, final band, and scattering mechanism will require establishing a mesh tailored to the unique threshold involved. The mesh used can vary from one scattering rate to another. A variable mesh, carefully specified in this way, allows a simple lincar interpolation to be used, while accurately representing the scattering rate near threshold, where it is apt to be most rapidly varying. Since the threshold depends only on energy, and is independent of the initial trajectory, the same energy mesh is used for each of the assorted initial trajectories for which the tabulation is performed.

The Brillouin zone, having cubic symmetry, may be represented by an irreducible zone, 1/48 of the full Brillouin zone in size, and bounded by rays in the dircetions [001], [111], and [101]. If the scattering rates, considered as a function of initial trajectory at a fixed energy, are known for all directions within the irreducible zone, then they may be found for any trajectory by the use of cubic symmetry operations. This principle is used to reduce the number of assorted trajectories required in the tabulation, it being sufficient to consider trajectories in the irreducible zone, only. For deformation potential acoustic and optical phonon scattering, the scattering rate anisotropy is low enough that the trajectories used in the rate tabulation may be restricted to the [001], [111], and [101] directions, which form the corners of the irreducible zone. Rates for other trajectories within the irreducible zone are obtained by using a bilinear interpolation on the tabulated rates at the three corners.

After the scattering mechanism and final band have been determined, the Monte Carlo must determine the final trajectory, to complete the simulation of a scattering event. This is the third area in which the present Monte Carlo method must deal with an aspect of the anisotropy associated with the valence band. The final trajectory is chosen probabilistically from a two-dimensional distribution in the two angles which determine the final trajectory. This distribution is proportional to the differential scattering rate associated with the initial state. The differential scattering rate, $d W / d \Omega^{\prime}$, is the integrand of the two-dimensional integral which is evaluated to give the scattering rate

$$
W=\int \frac{d W}{d \Omega^{\prime}} d \Omega^{\prime},
$$

where, as stated before, the integration is over the constant final energy surface. These distributions are calculated as needed, during the operation of the Monte Carlo.

The Monte Carlo method developed for this work can be used either to simulate a single particle's motion or to simulate a large number or ensemble of particles. The objectives and data collected differ in the two cases. To calculate the velocity-field characteristics, single particle simulations are done. During the simulation, the total distance and time traveled are recorded. After completion of the simulation, the ratio yields the drift velocity. To calculate diffusion, the en- semble mode of simulation is used. In this case, the average distance and the average of the square of the distance are recorded as functions of time, the average being over the large number of particles. After completion of the simulation, the diffusion coefficient is calculated as: ${ }^{23}$

$$
D=\frac{1}{2} \frac{d}{d t}\left(\left\langle x^{2}\right\rangle-\langle x\rangle^{2}\right) .
$$

The derivative must be taken away from the initial part of $\left\langle x^{2}\right\rangle-\langle x\rangle^{2}$, which is nonlinear. Of interest in the present work is the Ohmic mobility, which is related to the diffusion coefficient $D$ by the Einstein relation

$$
\mu=\frac{q D}{k_{B} T} .
$$

In pure materials, which are only considered in this work, the chemical potential tends to the middle of the band gap as temperature decreases, and therefore the Einstein relation, as given in Eq. (14), remains valid over the temperature range studied here. The appropriate derivation may be found in Ref. 24.

\section{PROCEDURE}

For both silicon and germanium, somewhat of a range of material parameters may be found in the published literature. In as much as these are input to a transport calculation, a corresponding range in calculated results may be expected. The most significant variations in material parameters are to be found in (a) band structure parameters: $\gamma_{1}, \gamma_{2}, \gamma_{3}, \Delta_{0}$, (b) elastic stiffness constants: $c_{11}, c_{12}, c_{44}$, and (c) deformation potentials: $a, b, d$. Many values for the deformation potential $a$, corresponding to hydrostatic compression, may be found tabulated in Ref. 25. A range of values found for the other parameters are given in Refs. 5,26-29 (band structure parameters), Refs. 5,30,31 (elastic stiffness constants) and Refs. 5,25,31-36 (deformation potentials $b$ and $d$ ).

Both the room-temperature value of hole mobility in silicon and germanium, and the temperature coefficients have been reported over a range. The coefficients are $M$ and $\alpha$, as in

$$
\mu(T)=M T^{-\alpha}
$$

with $T$ in degrees Kelvin. A range of values of $M$ and $\alpha$ are given in Refs. 37-40.

The first part of this work consisted of making a comparison of the Monte Carlo results with those of Szmulowicz. To do this, the same material parameters for silicon and germanium were used as reported in Ref. 7, which will be identified as parameter set $A$ (described below) with an optical phonon deformation potential value of $d_{n}=29.3 \mathrm{eV}$ for silicon and $d_{0}=40.0 \mathrm{eV}$ for germanium. Room-temperature conduction mobility and the temperature dependence of the mobility were obtained from the Monte Carlo calculated diffusion coefficients for both materials at zero electric field and compared with the mobility values reported by Szmulowicz. These mobilities are zero-field mobilities and therefore are independent of any particular field orientation. 
TABLE 1. Silicon material parameter sets used in this work. All parameters are for room temperature, unless otherwise noted.

\begin{tabular}{|c|c|c|c|c|c|}
\hline Parameter & & Symb. & Set $A^{a}$ & Set $\mathbf{B}^{\mathbf{a}}$ & Set $C^{a}$ \\
\hline Lattice constant $\AA$ & $(77 \mathrm{~K})$ & $a_{0}$ & $5.43086(41)$ & $\begin{array}{l}5.43095(42) \\
5.42701(43)\end{array}$ & $\begin{array}{l}\text { same as B } \\
\text { same as B }\end{array}$ \\
\hline$K-L$ parameter & & $\begin{array}{l}\gamma_{1} \\
\gamma_{2} \\
\gamma_{3}\end{array}$ & $\begin{array}{l}4.27(27,28) \\
0.315(27,28) \\
1.46(27,28)\end{array}$ & $\begin{array}{l}4.22(26) \\
0.39(26) \\
1.44(26)\end{array}$ & $\begin{array}{l}\text { same as B } \\
\text { same as B } \\
\text { same as B }\end{array}$ \\
\hline $\begin{array}{l}\text { Spin-orbit splitting }(\mathrm{eV}) \\
\text { Stiffness coeff. }\left(10^{11} \mathrm{dyn} / \mathrm{cm}^{2}\right)\end{array}$ & $(77 \mathrm{~K})$ & $\begin{array}{l}\Delta_{0} \\
c_{11}\end{array}$ & $\begin{array}{l}0.044(44) \\
16.56(31) \\
\ldots\end{array}$ & $\begin{array}{l}\text { same as A } \\
16.577(30) \\
16.722(30)\end{array}$ & $\begin{array}{l}\text { same as B } \\
\text { same as B } \\
\text { same as B }\end{array}$ \\
\hline & $\begin{array}{l}(77 \mathrm{~K}) \\
(77 \mathrm{~K})\end{array}$ & $\begin{array}{l}c_{12} \\
c_{44}\end{array}$ & $\begin{array}{l}6.39(31) \\
\ldots \\
7.95(31) \\
\ldots\end{array}$ & $\begin{array}{l}6.399(30) \\
6.497(30) \\
7.962(30) \\
8.036(30)\end{array}$ & $\begin{array}{l}\text { same as B } \\
\text { same as B } \\
\text { same as B } \\
\text { same as B }\end{array}$ \\
\hline Density $\left(\mathrm{g} / \mathrm{cm}^{3}\right)$ & & $\rho$ & $2.328(41)$ & $2.331(30)$ & same as B \\
\hline Optical phonon energy $(\mathrm{eV})$ & & $\hbar \omega_{0}$ & $0.063(41)$ & $0.0628(45)$ & same as $B$ \\
\hline Deformation pot. (eV) & & $\begin{array}{l}a \\
b \\
d\end{array}$ & $\begin{array}{l}2.1(25) \\
-2.2(25) \\
-5.3(25)\end{array}$ & $\begin{array}{l}\text { same as A } \\
\text { same as A } \\
\text { same as A }\end{array}$ & $\begin{array}{l}\text { same as B } \\
-1.5(31) \\
-3.4(31)\end{array}$ \\
\hline
\end{tabular}

${ }^{\mathrm{a}}$ Numbers in parentheses are references.

The uncertainty in the obtained diffusion coefficient is obtained from the $90 \%$ confidence interval about the mean of the three values $D_{x x}, D_{y y}$, and $D_{z z}$, using the $t$ distribution with two degrees of freedom. The uncertainty in the mobility is directly proportional to this, through the Einstein relation.

The second part of this work consisted of making a determination of the best values of $d_{0}$ for each semiconductor, considering a range of material parameters for each. For each semiconductor, three sets of material parameters were considered:

(1) Those used by Szmulowicz ${ }^{7}$ (except for $d_{0}$, which was to be determined). This is to be referred to as parameter set $\mathrm{A}$.

(2) A set which differs from the above primarily in the band structure and elastic stiffness constants; deformation potentials $a, b$, and $d$ are kept the same as in set $\mathrm{A}$. This is to be referred to as parameter set $B$.

(3) A set that differs from set B only in the values of deformation potentials $b$ and $d$. 'I'his is to be referred to as parameter set $\mathrm{C}$.

The spccific values of all parameters in sets $\mathrm{A}, \mathrm{B}$, and $\mathrm{C}$ are given in Table I (silicon) and Table II (germanium).

The determination of the best $d_{0}$ for each parameter set, in each semiconductor, is carried out in the following way. First, room-temperature mobilities are calculated for several values of $d_{0}$ spanning a range of about $10 \mathrm{eV}$. A linear leastsquares fit is made to the $\mu$ versus $d_{0}$ data. A value of $d_{0}$ is interpolated from this fit, which corresponds to a mobility equal to that reported in the literature for the given semiconductor. For silicon, the value of the mobility, taken from Ref.

TABLE II. Germanium material parameter sets used in this work. All parameters are for room temperature, unless otherwise noted.

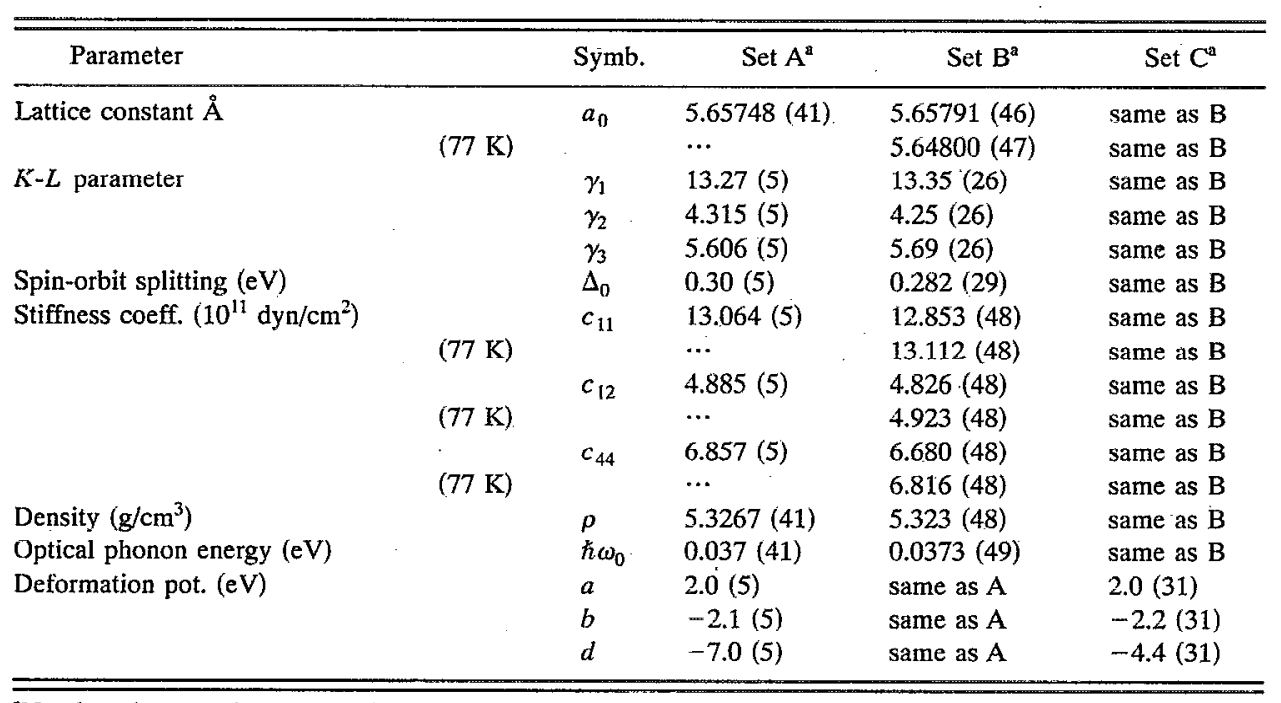

${ }^{a}$ Numbers in parentheses are references: 


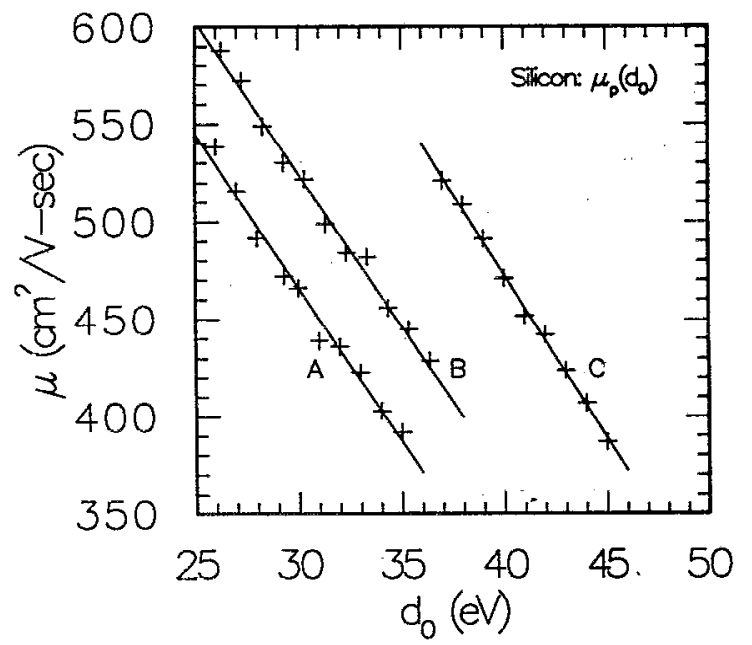

FIG. 1. Dependence of calculated silicon room-temperature ohmic mobility on optical phonon deformation potential, $d_{0}$, for each material parameter set A, B, and C. Straight lines are linear least-squares fits.

38 , is $480 \mathrm{~cm}^{2} / \mathrm{V} \mathrm{s}$. For germanium, the value of the mobility, taken from Ref. 40 , is $2060 \mathrm{~cm}^{2} / \mathrm{V} \mathrm{s}$. This gives the best $d_{0}$ for the given parameter set. Next, the relative merits of the different parameter sets are examined by calculating the temperature dependence of the mobility. For each parameter set, using the associated best $d_{0}$ value, the mobility is calculated at several values of temperature. The temperature is chosen over a range in which both acoustic and optical phonon scattering occur. This is $150-300 \mathrm{~K}$ for silicon and $100-300 \mathrm{~K}$ for germanium. A linear least-squares fit is made to the $\log \mu$ versus $\log T$ data over this range, allowing extraction of the temperature coefficients $M$ and $\alpha$, which are defined by the relation $\mu=M T^{-\alpha}$. Comparing the value of $\alpha$ with values given in the literature, gives an indication of which parameter set is most appropriate, along with the associated best value of $d_{0}$. Uncertainties in $M$ and $\alpha$ are obtained by allowing each value of mobility to vary within its range of uncer-

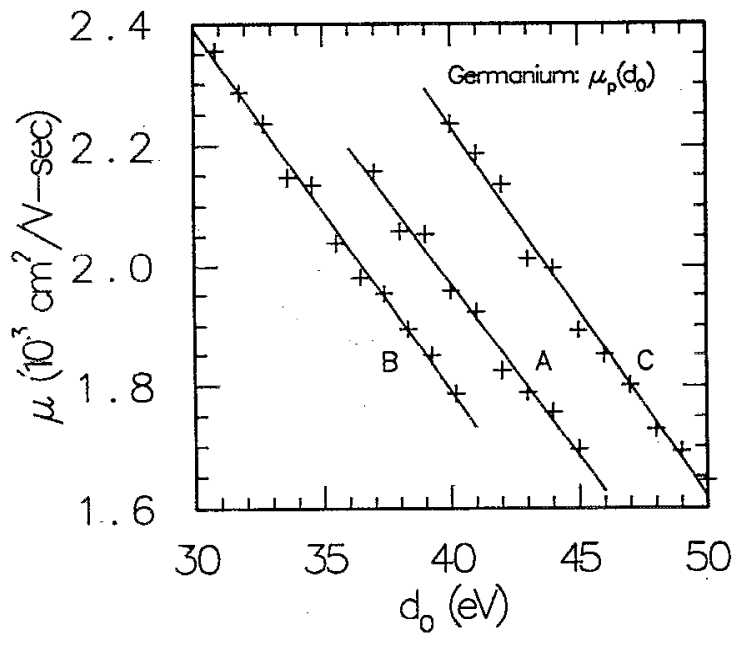

FIG. 2. Dependence of calculated germanium room-temperature ohmic mobility on optical phonon deformation potential $d_{0}$ for each material parameter set $A, B$, and $C$. Straight lines are linear least-squares fits.
TABLE III. Best values of optical phonon deformation potential $d_{0}$ determined by Monte Carlo, for cach parameter set in silicon and germanium. For these parameters, the room-temperature mobility is calculated to be 480 $\mathrm{cm}^{2} / \overline{\mathrm{V}} \mathrm{s}(\mathrm{Si})$ and $2060 \mathrm{~cm}^{2} / \mathrm{V} \mathrm{s}(\mathrm{Ge})$.

\begin{tabular}{lccc}
\hline \hline & \multicolumn{3}{c}{$d_{0}(\mathrm{eV})$} \\
\cline { 2 - 4 } Material & Set $\mathrm{A}$ & Set B & Set C \\
\hline $\mathrm{Si}$ & $29.1 \pm 0.7$ & $32.9 \pm 0.4$ & $39.6 \pm 0.5$ \\
$\mathrm{Ge}$ & $38.4 \pm 1.2$ & $35.5 \pm 0.4$ & $42.8 \pm 0.5$ \\
\hline \hline
\end{tabular}

tainty and determining the resulting range in $\alpha$ and $M$. Further examination of the different parameter sets is made by carrying out simulations of high field transport at room temperature. Here, the velocity-field data are obtained, using the different parameter sets.

\section{RESULTS}

First, comparison was made with the results of Szmulowicz. ${ }^{7}$ Using material parameter sets A, and optical phonon deformation potential values of $d_{0}=29.3 \mathrm{eV}$ for silicon and $d_{0}=40.0 \mathrm{eV}$ for germanium, the Monte Carlo calculations give the following room-temperature mobilities: $\mu_{\mathrm{Si}}=(472 \pm 12) \mathrm{cm}^{2} / \mathrm{V} \mathrm{s}$ and $\mu_{\mathrm{Ge}}=(1957 \pm 33) \mathrm{cm}^{2} / \mathrm{V} \mathrm{s}$. Over the temperature range of $150-300 \mathrm{~K}$, the calculated silicon mobility can be fit to $\mu=M(T / \text { Kelvin })^{-\alpha}$, with $M=5.76$ $\times 10^{8} \mathrm{~cm}^{2} / \mathrm{V} \mathrm{s}$ and $\alpha=2.46 \pm 0.09$. Over the temperature range of $100-300 \mathrm{~K}$, the calculated germanium mobility can be fit with $M=3.41 \times 10^{8} \mathrm{~cm}^{2} / \mathrm{V} \mathrm{s}$ and $\alpha=2.11 \pm 0.04$. In the work of Szmulowicz, using the same material parameters, the silicon and germanium room-temperature mobilities are $505 \mathrm{~cm}^{2} / \mathrm{V} \mathrm{s}$ and $2060 \mathrm{~cm}^{2} / \mathrm{V} \mathrm{s}$, respectively. Szmulowicz presents the temperature exponents $\alpha$ as varying with temperature, but having average values of 2.4 (silicon) and 2.3 (germanium). The agreement between the two methods is fairly close for the room-temperature mobilities in both materials and the temperature exponent in silicon. The tempera-

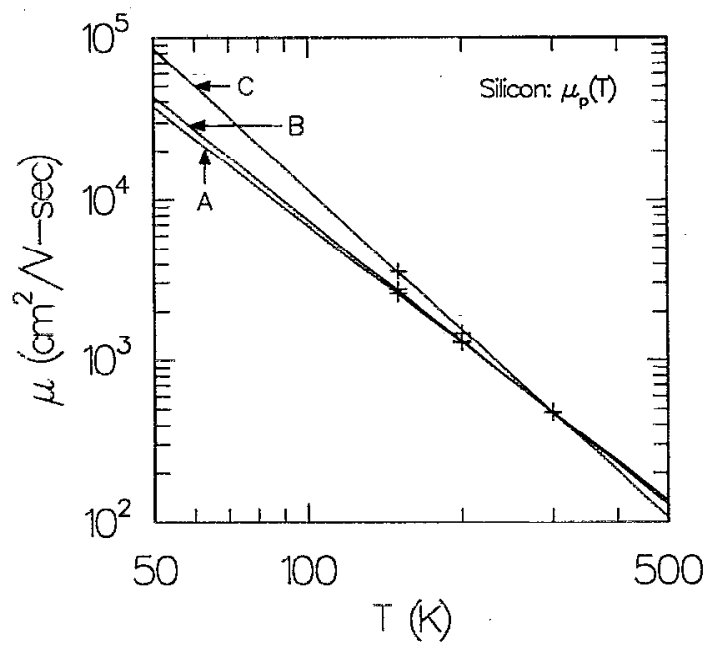

FIG. 3. Dependence of calculated silicon ohmic mobility on temperature, for each material parameter set A, B, and C. Straight lines are linear leastsquares fits to $\log \mu$ vs $\log T$. 


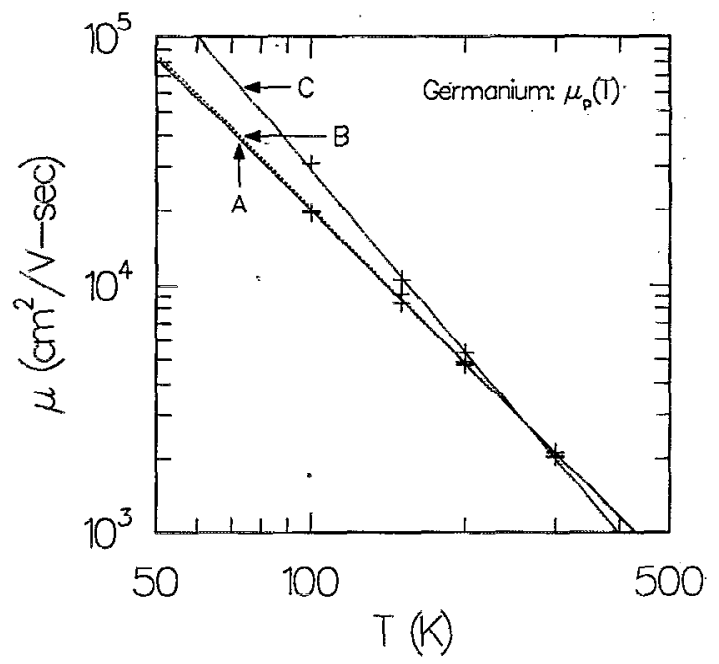

FIG. 4. Dependence of calculated germanium ohmic mobility on temperature, for each material parameter set A, B, and C. Straight lines are linear least-squares fits to $\log \mu$ vs $\log T$.

ture exponent for germanium has a greater discrepancy, which amounts to 0.1 when considering the upper limit of $\alpha=2.11 \pm 0.04$ from the Monte Carlo calculation and the lower limit of $\alpha=2.3 \pm 0.05$ from Szmulowicz' work.

Results of the calculation of the room-temperature mobilities as functions of the optical phonon deformation potential $d_{0}$ for each of the three material parameter sets in both silicon and germanium are shown in Figs. 1 and 2. From linear least-squares fits on these results, the best values of $d_{0}$ are determined as given in Table III.

Results of the calculation of the mobilities as functions of temperature, using the determined best values of $d_{0}$ for each parameter set, are shown in Figs. 3 and 4. Linear leastsquares fits are applied to $\log \mu$ versus $\log T$ to obtain the temperature coefficients $M$ and $\alpha$. These are presented in Table IV.

Finally, the results of the high-field transport calculations for each parameter set, in both materials, were obtained using an electric field oriented in the [100] direction. In previous work, ${ }^{16}$ good agreement has been found between the calculations with parameter set $\mathrm{C}$ and experimental data. In the present work, no significant dependence of the $v-E$ characteristics on the variation in material parameters was found.

TABLE IV. Temperature coefficients of the mobility determined from linear least-squares fits $\log \mu=\log M-\alpha \log T$.

\begin{tabular}{lccc}
\hline & & $\log \left(\frac{M}{\mathrm{~cm}^{2} / \mathrm{Vs}}\right)$ & $M\left(\mathrm{~cm}^{2} / \mathrm{V} \mathrm{s}\right)$ \\
\hline Material & $\alpha$ & $8.70 \pm 0.16$ & $4.98 \times 10^{8}$ \\
Si, set A & $2.43 \pm 0.07$ & $8.91 \pm 0.12$ & $8.22 \times 10^{8}$ \\
set B & $2.52 \pm 0.05$ & $9.86 \pm 0.24$ & $7.25 \times 10^{9}$ \\
set C & $2.90 \pm 0.10$ & $8.40 \pm 0.10$ & $2.51 \times 10^{8}$ \\
Ge, set A & $2.05 \pm 0.04$ & $8.46 \pm 0.07$ & $2.89 \times 10^{8}$ \\
set B & $2.08 \pm 0.03$ & $9.34 \pm 0.19$ & $2.20 \times 10^{9}$ \\
set C & $2.44 \pm 0.08$ & & \\
\hline \hline
\end{tabular}

\section{DISCUSSION}

In this paper we have shown that the Monte Carlo method can be used to study the problem of Ohmic transport of holes in $\mathrm{Si}$ and $\mathrm{Ge}$, with full consideration given to the band structure complexity. With the inclusion of appropriate scattering mechanisms, the method presented here can be extended to the study of Ohmic and high-field hole transport in other semiconductors.

We have also seen that small variations in the optical phonon deformation potential, $d_{0}$, have a quantitative effect on the temperature dependence of Ohmic hole mobility. However, there is sufficient uncertainty at present in the valence-band structure parameters, the valence-band acoustic deformation potentials, and the mcasured holc mobilitics that one can only obtain $d_{0}$ within a certain degree of uncertainty.

\section{ACKNOWLEDGMENT}

This work was supported by the U.S. Air Force (Grant No. AFOSR-91-0349).

${ }^{1}$ G. E. Pikus and G. L. Bir, Sov. Phys. Solid State 1, 1502 (1959).

${ }^{2}$ G. L. Bir and G. E. Pikus, Sov. Phys. Solid State 2, 2039 (1961).

${ }^{3}$ G. L. Bir and G. E. Pikus, Symmetry and Strain Induced Effects in Semiconductors (Wiley, New York, 1974).

${ }^{4}$ M. Tiersten, IBM J. Res. Dev. 5, 122 (1961).

${ }^{5}$ M. Tiersten, J. Phys. Chem. Sol. 25, 1151 (1964).

${ }^{6}$ P. Lawaetz, Phys. Rev. 174, 867 (1968).

${ }^{7}$ F. Szmulowicz, Phys. Rev. B 28, 5943 (1983).

${ }^{8}$ F. Meseguer, J. C. Merle, and M. Cardona, Solid State Commun. 43, 511 (1982).

${ }^{9}$ F. L. Madarasz and F. Szmulowicz, Phys. Rev. B 24, 4611 (1981).

${ }^{10}$ F. L. Madarasz, J. E. Lang, and F. Szmulowicz, J. Electrochem. Soc. 128, 2692 (1981).

${ }^{11}$ F. Szmulowicz and F. L. Madarasz, Phys. Rev, B 26, 2101 (1982).

${ }^{12}$ F. Szmulowicz and F. L. Madarasz, Phys. Rev. B 27, 2605 (1983).

${ }^{13}$ F. Szmulowicz and F. L. Madarasz, Phys. Rev. B 27, 6279 (1983).

${ }^{14}$ F. Szmulowicz, Appl. Phys. Lett. 43, 485 (1983).

${ }^{15} \mathrm{~J}$. M. Hinckley, Ph.D. thesis, University of Michigan, Ann Arbor, MI, 1990.

${ }^{16}$ J. M. Hinckley and J. Singh, Phys. Rev. B 41, 2912 (1990).

${ }^{17} \mathrm{~J}$. M. Hinckley and J. Singh (unpublished).

${ }^{18}$ G. Dresselhaus, A. F. Kip, and C. Kittel, Phys. Rev. 98, 368 (1955).

${ }^{19} \mathrm{~J}$. M. Lütinger, Phys. Rev. 102, 1030 (1956).

${ }^{20}$ E. M. Conwell and M. O. Vassell, Phys. Rev. 166, 797 (1968).

${ }^{21}$ M. Costato, G. Gagliani, and L. Reggiani, Lett. Nuovo Cimento 4, 171 (1972).

${ }^{22}$ H. Ehrenreich and A. W. Overhauser, Phys. Rev. 104, 331 (1956).

${ }^{23}$ C. Jacoboni and L. Reggiani, Rev. Mod. Phys. 55, 645 (1983).

${ }^{24}$ R. A. Smith, Semiconductors, 2nd ed. (Cambridge University Press, New York, 1978).

${ }^{25} \mathrm{M}$. Costato and L. Reggiani, Lett. Nuovo Cimento 4, 848 (1970).

${ }^{26}$ P. Lawaetz, Phys. Rev. B 4, 3460 (1971).

${ }^{27}$ H. Nakagawa and S. Zukotynski, Can. J. Phys. 55, 1485 (1977).

${ }^{28} \mathrm{H}$. Nakagawa and S. Zukotynski, Can. J. Phys. 56, 364 (1977).

${ }^{29}$ S. H. Groves, C. R. Pidgeon, and J. Feinleib, Phys. Rev. Lett. 17, 643 (1966).

${ }^{30}$ H. J. McSkimin and P. Andreatch, J. Appl. Phys. 35, 2161 (1964).

${ }^{31}$ J. D. Wiley, Solid State Commun. 8, 1865 (1970).

32 J. C. Hensel and G. Feher, Phys. Rev. 129, 1041 (1963).

${ }^{33}$ I. Balslev, Phys. Rev. 143, 636 (1966).

${ }^{34}$ I. Goroff and L. Kleinman, Phys. Rev. 132, 1080 (1963).

${ }^{35}$ S. Riskaer, Phys. Rev. 152, 845 (1966).

${ }^{36}$ I. Balslev, Phys. Rev. 177, 1173(1969).

${ }^{37}$ F. J. Morin and J. P. Maita, Phys. Rev. 96, 28(1954).

${ }^{38}$ G. W. Ludwig and K. L. Watters, Phys. Rev. 101, 1699(1956).

${ }^{39}$ F. J. Morin, Phys. Rev. 93, 62 (1954).

${ }^{40}$ D. M. Brown and R. Bray, Phys. Rev. 127, 1593(1962).

${ }^{41}$ S. M. Sze, Physics of Semiconductor Devices (Wiley, New York, 1981). 
${ }^{42}$ K. Godwod, R. Kowalczyk, and Z. Szmid, Phys. Status Solidi A 21, 227(1974).

${ }^{43}$ W. M. Yin and R. J. Paff, J. Appl. Phys. 45, 1456(1973).

${ }^{44}$ S. Zwerdling, K. J. Button, B. Lax, and L. M. Roth, Phys. Rev. Lett. 4, $173(1960)$.
${ }^{45}$ B. N. Brockhouse, Phys. Rev. Lett. 2, 256(1959).

${ }^{46}$ J. F. C. Baker and M. Hart, Acta Cryst. A 31, 364(1975).

${ }^{47}$ H. P. Singh, Acta Cryst. A 24, 469(1968).

${ }^{48}$ H. J. McSkimin and P. Andreatch, J. Appl. Phys. 34, 651(1963).

${ }^{49}$ G. Nilsson and G. Nelin, Phys. Rev. B 6, 3777(1972). 